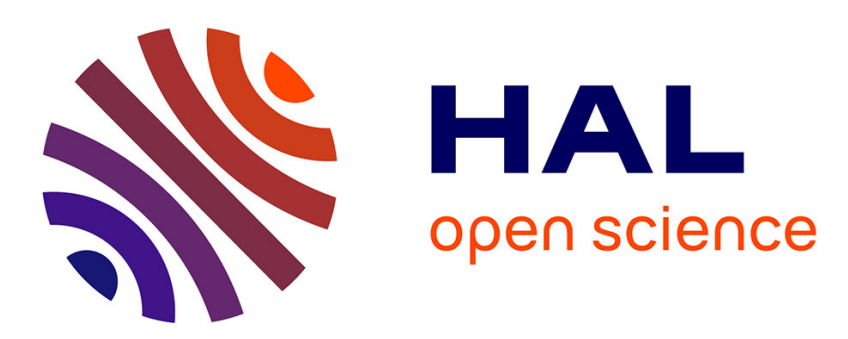

\title{
La relation entre l'épreuve de turbidité quantifiée et les propriétés du lait U.H.T. indirect
}

\author{
J. Mottar, R. Moermans
}

\section{To cite this version:}

J. Mottar, R. Moermans. La relation entre l'épreuve de turbidité quantifiée et les propriétés du lait U.H.T. indirect. Le Lait, 1983, 63 (623_624), pp.43-57. hal-00928965

\section{HAL Id: hal-00928965 https://hal.science/hal-00928965}

Submitted on 1 Jan 1983

HAL is a multi-disciplinary open access archive for the deposit and dissemination of scientific research documents, whether they are published or not. The documents may come from teaching and research institutions in France or abroad, or from public or private research centers.
L'archive ouverte pluridisciplinaire HAL, est destinée au dépôt et à la diffusion de documents scientifiques de niveau recherche, publiés ou non, émanant des établissements d'enseignement et de recherche français ou étrangers, des laboratoires publics ou privés. 


\title{
La relation entre l'épreuve de turbidité quantifiée et les propriétés du lait U.H.T. indirect
}

\author{
par \\ J. MOTTAR* et R. MOERMANS**
}

\begin{abstract}
Rés u mé
Le rapport entre la turbidité mesurée par néphélométrie et quelques caractéristiques importantes du lait U.H.T., chauffé par la méhode indirecte, a été étudié. Les essais ont été effectués à l'échelle pilote, sans récupération de chaleur.

Les relations entre d'une part, le degré de turbidité et d'autre part : l'effet sporicide, la dénaturation des protéines lactosériques, la formation d'hydroxyméthylfurfural, la perte de lysine disponible et l'inactivation d'enzymes protéolytiques, peuvent être décrites par un modèle mathématique composé de deux droites avec un point d'intersection. Pour chaque facteur étudié ce point est situé aux environs d'un degré de turbidité moyen de 50 NTU. Au-dessous de cette valeur la pente des droites diminue significativement. Ceci signifie qu'à des turbidités plus faibles, des modifications minimes du degré de turbidité pourraient avoir un effet relativement considérable sur certaines caractéristiques importantes du lait.

En cas d'application d'une récupération de chaleur, il faut s'attendre à des modifications principalement de la relation entre la turbidité et l'effet sporicide. Pour les caractéristiques chimiques, les résultats expérimentaux sont transférables aux conditions pratiques.

Un lait U.H.T. chauffé par la méthode indirecte et dont la turbidité est égale à 50 NTU ou supérieure dans certaines limites est d'une qualité garantie acceptable, sans risque d'une efficacité stérilisatrice trop faible.
\end{abstract}

* Station Laitière de l'Etat, Melle. Centre de Recherches Agronomiques de l'Etat, Gand (Belgique).

** Bureau de Biométrie, Centre de Recherches Agronomiques de l'Etat, Gand (Belgique). 
Dans la pratique, ceci veut dire que lorsqu'on applique le procédé U.H.T. indirect, il faut limiter le degré de récupération de chaleur si l'on veut maintenir une différence de caractéristiques par rapport au lait stérilisé en deux étapes. Des turbidités suffisamment élevées peuvent être obtenues avec le lait U.H.T. chauffé par la méthode indirecte, sans risque d'insuffisance de conservabilité.

Mots clés :

Lait U.H.T. - Epreuve de turbidité quantifiée - Effet sporicide - Caractéris-

tiques chimiques.

\section{Su m m a ry}

THE RELATION BETWEEN THE QUANTIFIED TURBIDITY TEST AND THE PROPERTIES OF INDIRECT HEATED U.H.T. MILK

The relationship between the nephelometrically measured turbidity and some important characteristics of indirectly heated U.H.T. milk was investigated. The tests were carried out on a pilot scale, without heat recovery.

To describe the relationship between the turbidity and the milk characteristics such as sporicidal effect, whey protein denaturation, formation of hydroxymethylfurfural, loss of available lysine and inactivation of proteolytic enzymes, the proposed mathematical model is a two-phase lineair regression with a point of intersection. For each parameter this point is situated near the average turbidity value of $50 \mathrm{NTU}$. Beneath this value the slope of the regression line decreases significantly. This means that minor changes in turbidity may, at lower values, influence some important milk characteristics to a high degree.

When heat recovery is applied in the indirect U.H.T. system, modifications of the relationship are to be expected, mainly for the relation between the turbidity and the sporicidal effect. For the chemical characteristics, the experimental results are transferable to pratical conditions.

Indirectly heated U.H.T. milk with a turbidity of about $50 \mathrm{NTU}$, or higher within certain limits, guarantees an acceptable quality, without risk of a too less sterilizing effect.

Practically this means that the heat recovery level is to be limited in the indirect U.H.T. process, in order to maintain a characteristical distinction with two-stage sterilized milk. Sufficiently high turbidities may be obtained for indirectly heated U.H.T. milk without risk of a too short shelf life.

Key words:

U.H.T. milk - Quantified turbidity test - Sporicidal effect - Chemical characteristics. 


\section{INTRODUCTION}

Dans plusieurs pays, le lait U.H.T. est considéré comme différent du lait pasteurisé et du lait stérilisé en bouteille. Il est donc désirable de disposer d'une méthode suffisamment rapide et précise, permettant de caractériser le lait thermisé.

Le test d'Aschaffenburg, basé sur l'appréciation visuelle de la turbidité, a longtemps été unanimement accepté comme méthode adéquate pour distinguer le lait U.H.T. du lait stérilisé dans la bouteille (Pien, 1972).

L'introduction des installations de traitement U.H.T. indirect combiné avec un degré élevé de récupération de chaleur, a eu toutefois pour conséquence de ne plus permettre, dans certains cas, de distinguer le lait U.H.T. du lait stérilisé en deux étapes (Mottar et Naudts, 1979).

Une épreuve de turbidité quantifiée a été élaborée afin d'augmenter la précision de la méthode d'Aschaffenburg (Mottar et Naudts, 1981). Lechner (1982) croit pouvoir discerner certains inconvénients dans l'épreuve de turbidité. La teneur résiduelle du lait U.H.T. en protéines lactosériques non dénaturées dépend de la durée du traitement thermique. Selon cet auteur, des fluctuations de la teneur initiale en ces protéines pourrait aussi influer sur le degré de turbidité. Selon notre expérience, les facteurs susdits ne déterminent cependant pas le degré de turbidité tel qu'il est relevé par nephélométrie.

Considérant la simplicité et la rapidité d'exécution de l'épreuve de turbidité quantifiée, il semblait indiqué de rechercher en quelle mesure cette méthode peut fournir des informations sur un certain nombre de caractéristiques importantes du lait U.H.T. Notre attention s'est portée sur le lait U.H.T. traité par le procédé indirect. Il est établi, en effet, que les caractéristiques de ce lait peuvent être influencées à un tel point par le traitement thermique, qu'il n'existe plus, dans certains cas, de différence avec le lait stérilisé dans la bouteille (Mottar et Naudts, 1979). La question se pose de savoir comment le procédé indirect doit être mis en œuvre pour que les caractéristiques du produit final soient suffisamment différentes de celle du lait stérilisé en deux étapes et de savoir si ceci peut être contrôlé à l'aide de l'épreuve de turbidité.

\section{MATERIEL ET METHODES}

\section{Conditions de traitement}

Du lait cru de mélange réfrigéré avec une teneur en matière grasse entre 3,5 et $3,7 \%$ a été soumis à différents traitements U.H.T. 
dans une installation pilote APV, équipée d'un échangeur thermique à plaques dans sa section U.H.T. Un schéma de l'appareil est représenté dans la figure 1. La figure 2 donne un schéma de l'évolution

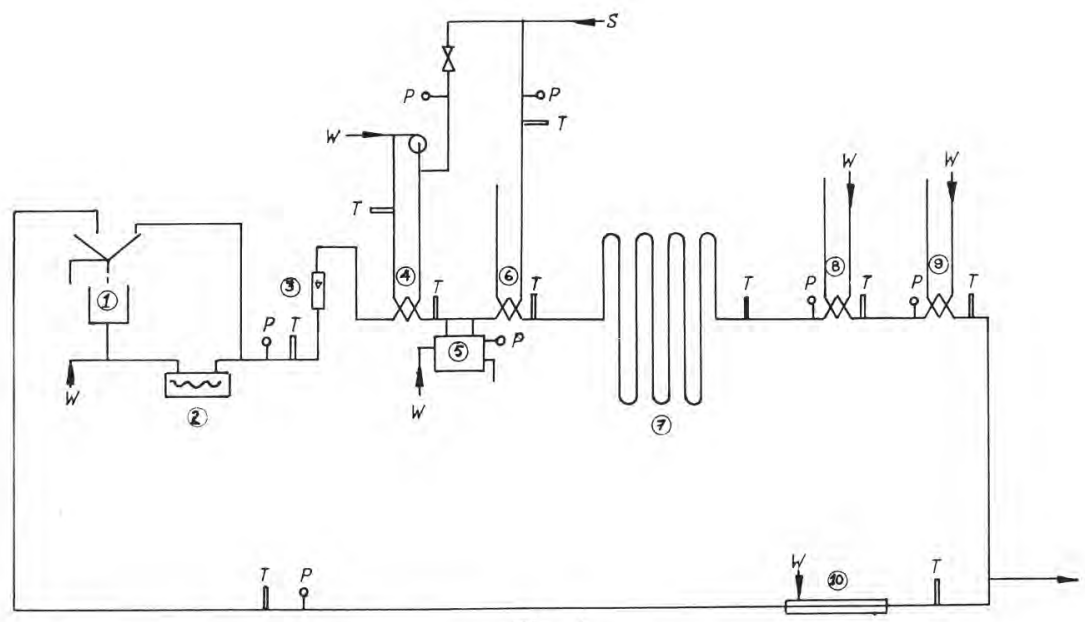

Fig. 1

Représentation schématique de l'appareil pilote U.H.T. indirect.

1. Réservoir.

2. Pompe à haute pression.

3. Indicateur de débit.

4. Section de préchauffage.

5. Homogénéiseur.

6. Section de stérilisation.

7. Section de séjour.
8.-9. Section de refroidissement.

10. Stérilisateur du circuit.

W. Eau.

S. Vapeur.

T. Thermocouple.

P. Manomètre.

de la température en fonction du temps. Les températures ultrahautes variaient entre 130 et $150^{\circ} \mathrm{C}$; les temps de séjour entre 2,5 et $45 \mathrm{~s}$. Le système ne comportait pas de récupération de chaleur. Ceci implique que le chauffage et le refroidissement se déroulent relativement vite, ce dont il faut tenir compte dans l'interprétation des résultats. Le lait a été préchauffé à $80^{\circ} \mathrm{C}$, puis homogénéisé en deux étapes sous des pressions de respectivement 21,6 et 4,9 MPa. Les temps de séjour désirés ont été instaurés par une incorporation adéquate de tuyaux en aval du chauffage à ultra-haute température. Les températures étaient contrôlées à l'aide de thermocouples.

Le lait de mélange employé dans ces essais était du type traité dans la pratique, à nombre de germes situé entre $2.10^{5}$ et $5.10^{5}$ par $\mathrm{ml}$.

Le lait U.H.T. était soutiré en conditions d'asepsie dans des bouteilles d'un litre, en polyéthylène, fermées en conditions de stérilité par un couvercle d'aluminium revêtu de polyéthylène. Les 


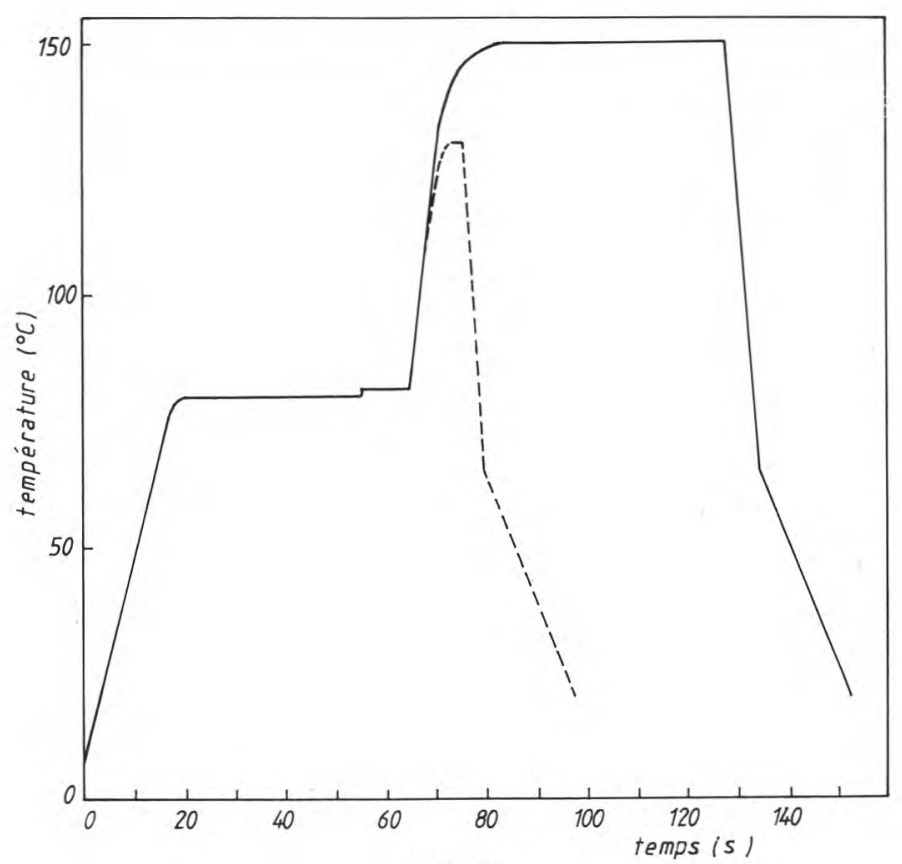

Fig. 2

Schéma de l'évolution de la température en fonction du temps du traitement U.H.T.

échantillons étaient stockés dans l'obscurité, à $5^{\circ} \mathrm{C}$, et analysés dans les deux semaines suivant la production.

\section{Détermination de l'effet sporicide}

L'effet sporicide a été déterminé selon la méthode de Galesloot telle que décrite par Mottar (1981) et avec usage d'une souche thermo-résistante de Bacillus subtilis 0125 (NIZO), ayant une valeur de résistance thermique $\mathrm{z}$ de $10,7^{\circ} \mathrm{C}$ pour l'intervalle de température entre 130 et $150^{\circ} \mathrm{C}$. Il fut veillé à ce que le nombre initial de spores du lait, avant le traitement thermique, s'élevât au moins à $10^{7}$ par $\mathrm{ml}$. Les teneurs en spores furent déterminées par repiquage sur Plate Count Agar, après chauffage du lait pendant $10 \mathrm{~min}$ à $80^{\circ} \mathrm{C}$.

\section{Méthodes d'analyse}

La détermination du degré de turbidité a été faite sur le filtrat obtenu en conditions standardisées, après précipitation du lait à l'aide de $20 \%$ de sulfate d'ammonium et réchauffement au bain-marie d'eau bouillante, comme décrit par Mottar et Naudts (1981). Le 
degré de turbidité a été mesuré au moyen d'un néphélomètre Fisher DRT-100 et les résultats ont été exprimés en unités de turbidité néphélométriques (NTU).

Les fractions azotées ont été extraites par la technique d'Aschaffenburg et Drewry (1959). L'azote a été dosé par la méthode micrométrique de Kjeldahl. La teneur en protéines lactosériques (WPN) a été calculée d'après la différence entre l'azote non caséinique (NCN) et l'azote non protéique (NPN). Le degré de dénaturation, exprimé en pourcentage des protéines lactosériques, a pu être calculé par comparaison de la teneur résiduelle en WPN du lait thermisé avec la teneur initiale.

La détermination de la teneur totale en hydroxyméthylfurfural (HMF) était basée sur la méthode de Keeney et Basette (1959). La concentration en HMF a été calculée selon l'équation de régression $\mathrm{Y}=87,5 \mathrm{X}-4,8(\mathrm{X}=$ la densité optique, mesurée à $443 \mathrm{~nm})$ et est exprimée en $\mu \mathrm{mol} / 1$. La lysine disponible a été mesurée selon la méthode de Carpenter modifiée, telle que décrite par Booth (1971).

L'activité protéolytique dans le lait a été mesurée par incubation anaérobique à $37^{\circ} \mathrm{C}$. Du thimerosal a été ajouté au lait, à raison de $0,01 \%$, en guise d'inhibiteur de croissance de micro-organismes (Noomen, 1975). L'azote non caséinique a été déterminé après 0, 3, 6 , 9 et 12 jours. Les activités protéolytiques ont été calculées par analyse de régression linéaire simple et exprimées comme augmentation en mg par $100 \mathrm{~g}$ de lait et par $24 \mathrm{~h}$, de l'azote non caséinique. L'activité résiduelle dans le lait thermisé a été exprimée en pourcentage, par rapport à l'activité du lait de départ.

\section{Méthodes mathématiques}

Les observations ont été décrites par le modèle mathématique :

$$
\begin{aligned}
& Y_{i}=a+b X_{i} \text { pour } X_{i} \leqslant X_{I} \\
& Y_{i}=a^{\prime}+b^{\prime} X_{i} \text { pour } X_{i} \geqslant X_{I}
\end{aligned}
$$

Dans les équations $\mathrm{Y}$ représente la turbidité et $\mathrm{X}$ respectivement l'effet sporicide, la dénaturation des protéines lactosériques, la teneur en HMF, les pertes de lysine et le degré d'inactivation d'enzymes protéolytiques; $\mathrm{X}_{\mathrm{I}}$ est le point d'intersection inconnu. Ce point est calculé tel que la somme des sommes des carrés des écarts soit minimale. Les deux droites ont été testées par une analyse de la variance. Les limites de confiance inverses au seuil 0,95 sur $\mathrm{X}_{\text {I }}$ ont été calculées en appliquant le théorème de Fieller, comme décrit par Finney (1978). Les limites de confiance sur la valeur moyenne de turbidité au niveau du point d'intersection $\left(\mathrm{Y}_{\max }\right.$. ; $\mathrm{Y}_{\min }$ ) ont été calculées en substituant les limites inverses dans les équations obtenues.

Pour les deux droites, les limites de confiance au seuil 0,95 sur les valeurs moyennes de turbidité ont été calculées, ainsi que les 
limites de confiance au seuil 0,95 sur les valeurs individuelles de turbidité pour les facteurs dénaturation de protéines lactosériques et teneur en HMF, selon Draper et Smith (1966).

\section{RESULTATS ET DISCUSSION}

\section{Relation entre la turbidité, la température et la durée du traitement U.H.T.}

La figure 3 reproduit les turbidités mesurées du lait pour les diverses combinaisons de température et de durée du traitement U.H.T. indirect. Chaque combinaison de température et de durée a été répétée 4 fois, de sorte que les résultats reproduits sont les moyennes de 4 observations. Dans la zone de températures de 130 à

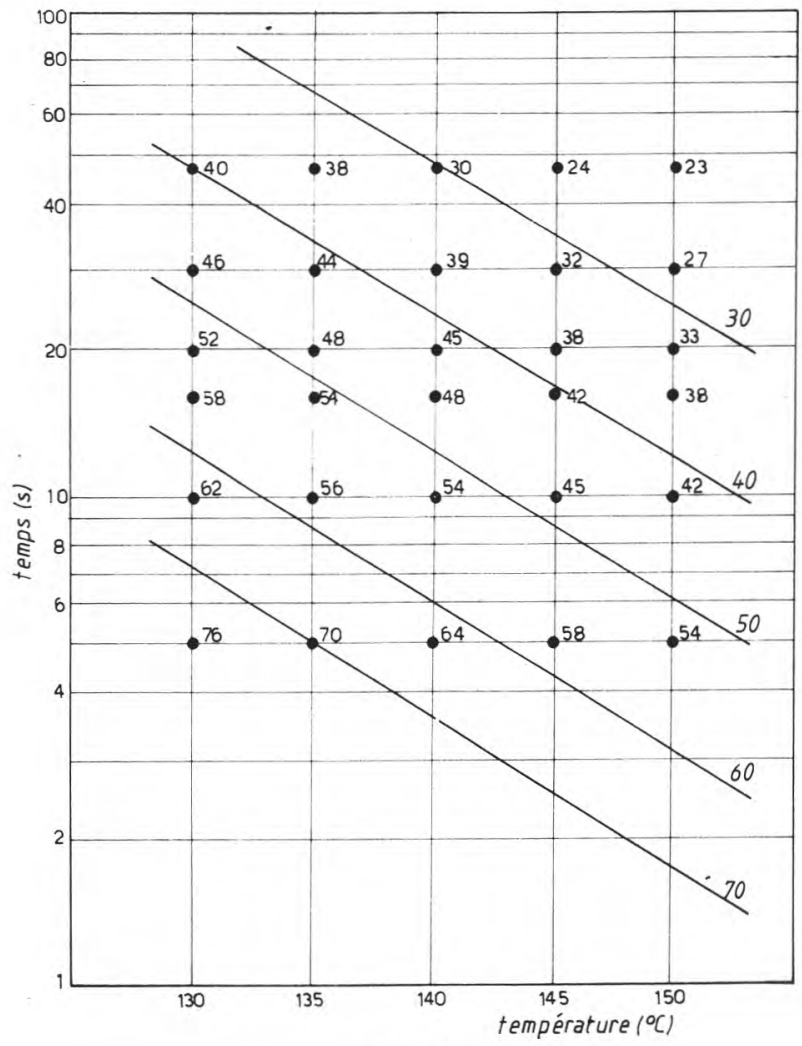

Fig. 3

Le degré de turbidité en fonction de la température et de la durée d'un traitement U.H.T. indirect. 
$150^{\circ} \mathrm{C}$, des limites linéaires de turbidité identique peuvent être déduites en un diagramme semi-logarithmique de températures et de durées. Les droites ont été obtenues par ajustement visuel.

Les ultra-hautes températures et durées de traitement requises pour obtenir la turbidité désirée peuvent être déduites de la figure, par extrapolation.

On peut déduire de la pente des droites que la valeur $\mathrm{z}$ est de $32,2^{\circ} \mathrm{C}$, ce qui est comparable à la valeur trouvée par Mottar, (1981) pour la dénaturation des protéines lactosériques $\left(28,6^{\circ} \mathrm{C}\right)$ et celles également déterminées par Agrawala et Reuter (1979). Pour un traitement U.H.T. avec des températures de préchauffage entre 70 et $80^{\circ} \mathrm{C}$ ces auteurs trouvaient des valeurs de résistance à la chaleur entre 28,7 et $37,7^{\circ} \mathrm{C}$.

Il est évident qu'une récupération croissante de chaleur fera baisser les températures, dans la zone U.H.T., et abrégera les durées de traitement U.H.T. pour aboutir à un même degré de turbidité.

\section{Relation entre la turbidité et l'effet sporicide}

Le degré de destruction des spores bactériennes sous l'effet du traitement thermique dépend du nombre de spores présentes dans le lait cru, de la thermorésistance de ces spores et du nombre de spores toléré dans le produit final. Une perte de production de $0,1 \%$ par développement de spores bactériennes est commercialement

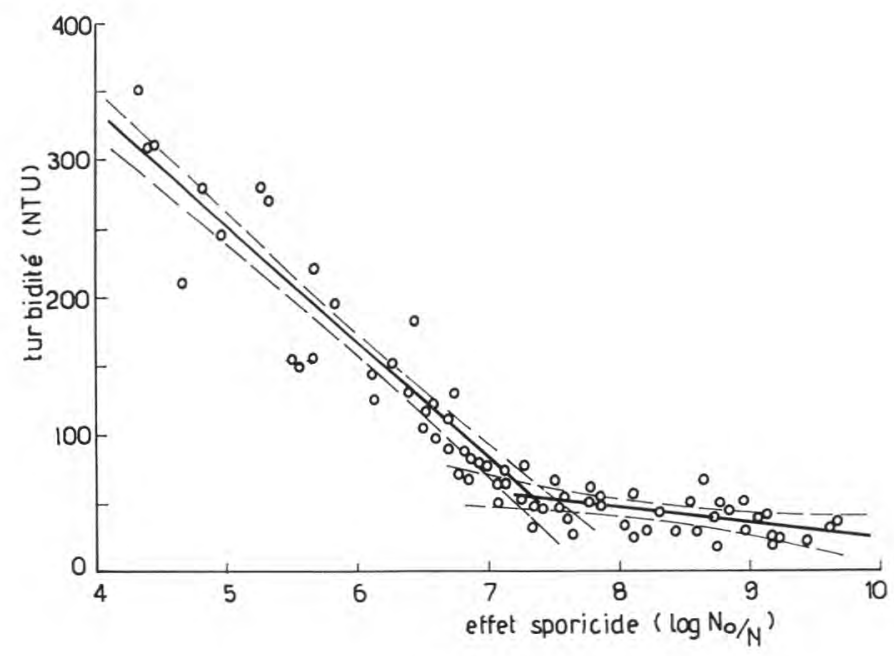

Fig. 4

Relation entre le degré de turbidité et l'effet sporicide $(---$ : limites de confiance au seuil 0,95 sur les valeurs moyennes de turbidité). 
acceptable (Franklin, 1970; Langeveld et al., 1976). Compte tenu d'une teneur maximale de 10 spores thermorésistantes par $\mathrm{ml} \mathrm{du}$ lait cru (Waes, 1976), un effet sporicide d'au moins 7 est requis pour maintenir la perte de production dans les limites acceptables. Il peut toutefois arriver que le lait thermisé exerce une action inhibitrice sur la germination et le développement de spores bactériennes (Franklin, 1970), ce qui réduit encore plus les pertes de production imputables au développement de spores. Enfin, toutes les spores présentes dans le lait n'ont pas la même thermorésistance que les spores de la souche de Bacillus subtilis que nous avons utilisée. Ceci permet de supposer que l'effet sporicide peut, en réalité, être notablement plus grand que celui déterminé au cours des expériences.

La figure 4 illustre la relation existant́ entre le degré de turbidité $(\mathrm{Y})$ et l'effet sporicide $(\mathrm{X})$ du traitement thermique. Les paramètres de cette relation sont :

\begin{tabular}{|c|c|c|c|c|c|c|c|}
\hline \multirow{2}{*}{ Equations } & \multirow{2}{*}{ Fcalc. } & \multirow{2}{*}{$P$} & \multirow{2}{*}{$\mathrm{r}$} & \multicolumn{3}{|c|}{ Intersection } & \multirow{2}{*}{$\begin{array}{l}\text { Erreur } \\
\text { stand. }\end{array}$} \\
\hline & & & & $(\mathrm{X}, \mathrm{Y})$ & $Y_{\min }$. & $Y_{\max }$ & \\
\hline $\begin{array}{l}\mathrm{Y}=673,47-84,71 \mathrm{X} \\
\mathrm{Y}=140,58-11,89 \mathrm{X}\end{array}$ & $\begin{array}{r}203,3 \\
30,5\end{array}$ & $\begin{array}{l}<0,001 \\
<0,001\end{array}$ & $\begin{array}{l}-0,93 \\
-0,64\end{array}$ & 7,$3 ; 53$ & 50 & 72 & 21,9 \\
\hline
\end{tabular}

Si l'on admet qu'une efficacité stérilisatrice de 7 est commercialement acceptable, on peut déduire de la figure 4, tenant compte des intervalles de confiance, que pour le lait U.H.T., traité par le procédé indirect, on peut obtenir des turbidités moyennes jusqu'à environ $92 \mathrm{NTU}$, sans risque d'une efficacité stérilisatrice trop faible.

Il convient de remarquer à ce propos que le degré de récupération de chaleur peut exercer une influence sur la destruction des spores et sur la turbidité. En se basant sur des différences en coefficient de température de traitements chimiques et bactériologiques, on peut admettre que pour atteindre un même degré de turbidité, on obtiendra une moins grande destruction de spores en cas de degré de récupération de chaleur croissant.

Les données obtenues ne peuvent pas être reportées intégralement sur les installations industrielles, entre autres à cause de l'existence d'un certain degré de récupération de chaleur, de différences en gradient de température entre le produit et le fluide de chauffage et aussi en raison de conditions de passage différentes du produit dans l'échangeur thermique. Les expériences doivent donc être recommencées pour chaque installation si l'on veut connaître exactement la relation entre la turbidité et l'effet sporicide.

Il est toutefois évident que la récupération de chaleur des installations U.H.T. de type indirect doit rester limitée si l'on veut 
atteindre l'effet sporicide minimal requis, tout en obtenant encore une turbidité suffisamment élevée.

Le nombre de spores de la matière première étant codéterminant de l'effet sporicide, il est conseillé, de ne traiter que des laits de bonne qualité bactériologique.

\section{Relation entre la turbidité et quelques réactions chimiques}

\subsection{Dénaturation des protéines lactosériques}

Les protéines lactosériques sont très vulnérables à la dénaturation sous l'effet de la chaleur. La détermination du degré de dénaturation fournit des informations sur l'intensité d'un traitement thermique.

Le rapport entre la turbidité (Y) et le degré de dénaturation de la fraction de protéines lactosériques (X) est reproduit dans la figure 5. Cette relation est décrite par les caractéristiques suivantes :

\begin{tabular}{c|c|c|c|c|c|c|c}
\hline & & & & \multicolumn{2}{|c|}{ Intersection } & Erreur \\
Equations & Fcalc. & $\mathrm{P}$ & $\mathrm{r}$ & $\mathrm{X}, \mathrm{Y})$ & $\mathrm{Y}_{\min .}$ & $\mathrm{Y}_{\max .}$ & \\
\hline $\mathrm{Y}=1493,98-21,17 \mathrm{X}$ & 73,5 & $<0,001$ & $-0,86$ & & & & \\
\hline $\mathrm{Y}=361,19-4,26 \mathrm{X}$ & 152,3 & $<0,001$ & $-0,82$ & 67,$0 ; 76$ & 68 & 103 & 24,0 \\
\hline
\end{tabular}

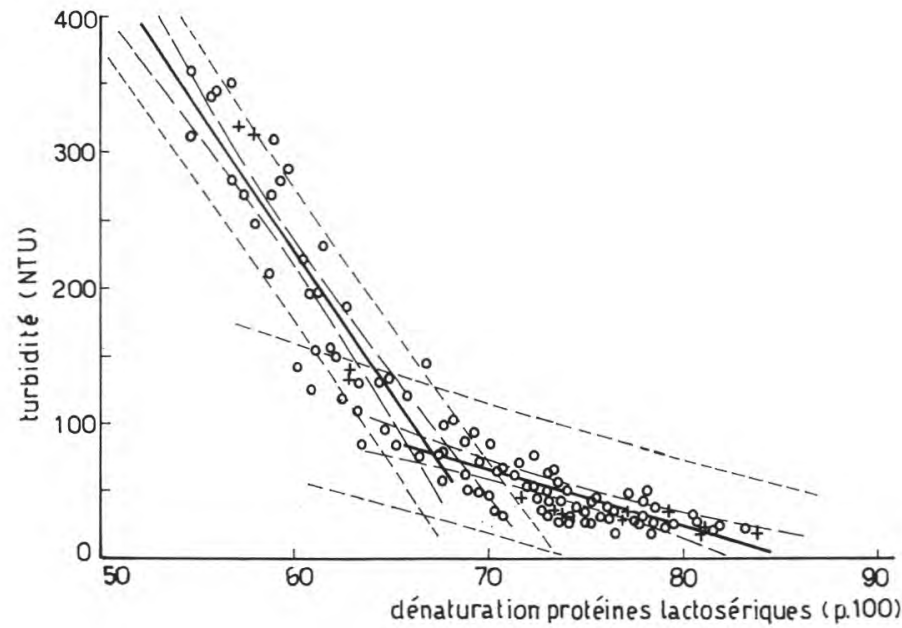

Fig. 5

Relation entre le degré de turbidité et la dénaturation des protéines lactosériques (- - : limites de confiance au seuil 0,95 sur les valeurs moyennes de turbidité ; ... - limites sur les valeurs individuelles; + : observations pratiques). 


\subsection{Formation d'hydroxyméthylfurfural}

La formation de HMF dépend dans une large mesure de l'intensité de la température à laquelle le lait est soumis (Mottar et Naudts, 1979).

Le rapport entre la turbidité (Y) et la teneur en HMF (X), mesurée aussitôt après le traitement thermique, est représenté dans la figure 6. La relation est caractérisée par :

\begin{tabular}{c|c|c|c|c|c|c|c}
\hline & & & & \multicolumn{2}{|c|}{ Intersection } & Erreur \\
Equations & Fcalc. & $\mathrm{P}$ & $\mathrm{r}$ & $\mathrm{X}, \mathrm{Y})$ & $\mathrm{Y}_{\mathrm{min} .}$ & $\mathrm{Y}_{\max .}$ & \\
\hline $\mathrm{Y}=440,42-53,95 \mathrm{X}$ & 19,7 & $<0,001$ & $-0,76$ & & & & \\
\hline $\mathrm{Y}=72,50-2,31 \mathrm{X}$ & 44,8 & $<0,001$ & $-0,64$ & 7,$1 ; 56$ & 54 & 89 & 30,7 \\
\hline
\end{tabular}

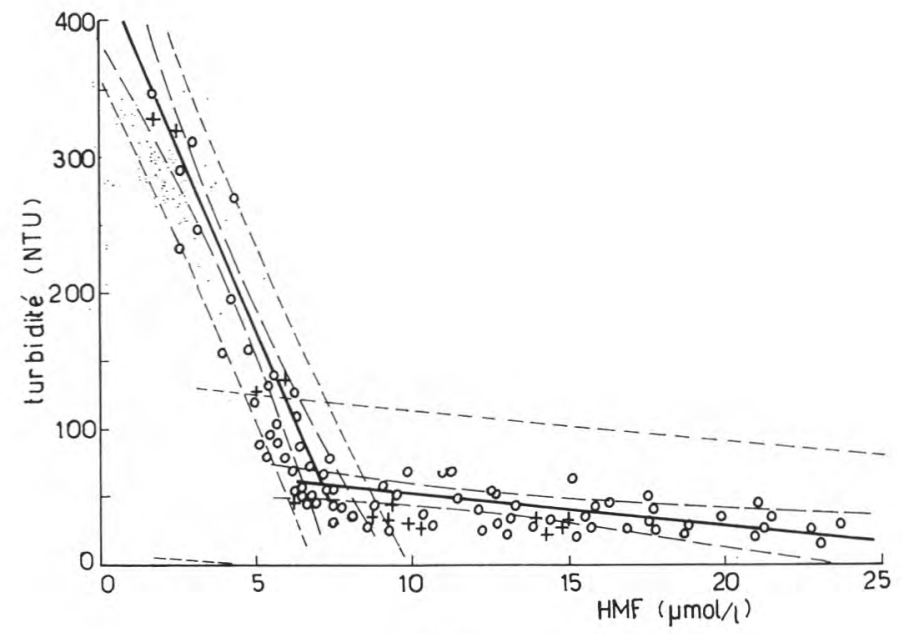

Fig. 6

Relation entre le degré de turbidité et la formation de HMF ( - - : limites de confiance au seuil 0,95 sur les valeurs moyennes de turbidité ; ... : limites sur les valeurs individuelles ; + : observations pratiques).

\subsection{Perte de lysine disponible}

La lysine est un acide aminé essentiel, qui ne peut être fourni à l'organisme que par l'alimentation. Le lait en est une source excel- 
lente. La lysine peut toutefois être impliquée dans la réaction de Maillard au cours de la thermisation du lait. Cet acide aminé n'étant plus disponible pour l'organisme, la valeur nutritive du lait s'en trouve diminuée.

Le rapport entre la turbidité $(\mathrm{Y})$ et les pertes en lysine disponible (X) est reproduit dans la figure 7, dont les caractéristiques sont :

\begin{tabular}{c|c|c|c|c|c|c|c}
\hline \multirow{2}{*}{ Equation } & Fcalc. & $\mathrm{P}$ & $\mathrm{r}$ & \multicolumn{2}{|c|}{ Intersection } & $\begin{array}{c}\text { Erreur } \\
\text { stand. }\end{array}$ \\
\hline $\mathrm{Y}=25,02-5,44 \mathrm{X}$ & 53,4 & $<0,001$ & $-0,80$ & $0 ; 75$ & 67 & 85 & 10,1 \\
\hline
\end{tabular}

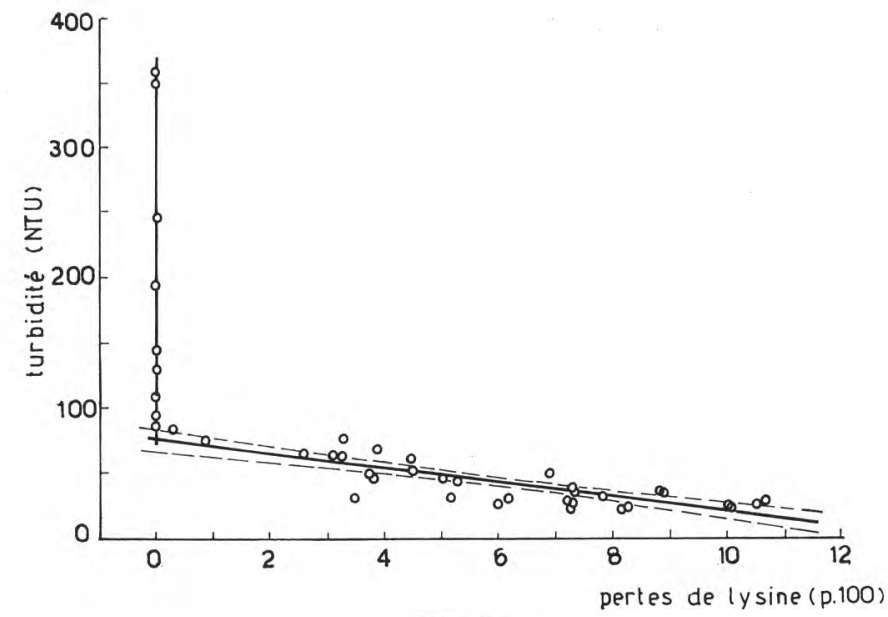

Fig. 7

Relation entre le degré de turbidité et les pertes en lysine disponible ( - - : limites de confiance au seuil 0,95 sur les valeurs moyennes de turbidité).

\subsection{Inactivation d'enzymes protéolytiques}

La présence d'enzymes protéolytiques thermostables dans le lait U.H.T. peut contribuer dans une mesure importante à l'apparition de défauts organoleptiques et physico-chimiques pendant la conservation. 
La figure 8 reproduit la relation entre le degré d'inactivation des enzymes protéolytiques $(\mathrm{X})$ et le degré de turbidité résultant $(\mathrm{Y})$. Ce rapport peut être décrit par :

\begin{tabular}{c|c|c|c|c|c|c|c}
\hline & \multirow{2}{*}{ Equations } & Fcalc. & $\mathrm{P}$ & $\mathrm{r}$ & \multicolumn{2}{|c|}{ Intersection } & $\begin{array}{c}\text { Erreur } \\
\text { stand. }\end{array}$ \\
\hline $\mathrm{Y}=1363,64-14,38 \mathrm{X}$ & 12,8 & $<0,01$ & $-0,80$ & 89,$3 ; 79$ & 16 & 139 & 20,5 \\
$\mathrm{Y}=508,92-4,81 \mathrm{X}$ & 199,5 & $<0,001$ & $-0,85$ & & & & \\
\hline
\end{tabular}

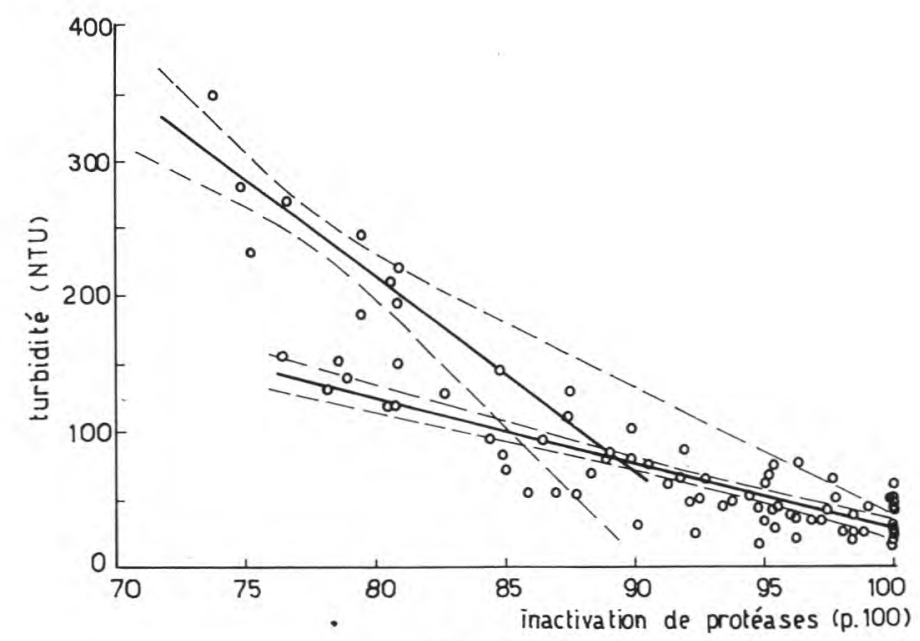

Fig. 8

Relation entre le degré de turbidité et l'inactivation de protéases ( $-\ldots$ - : limites de confiance au seuil 0,95 sur les valeurs moyennes de turbidité).

\subsection{Remarques générales à propos des relations constatées}

Les diverses relations entre la turbidité et les effets chimiques peuvent être décrites par deux droites avec un point d'intersection qui se situe en moyenne entre environ 50 et 80 NTU pour la valeur de turbidité. En règle générale, on peut admettre qu'en dessous d'un degré de turbidité moyen de 50 NTU, la pente des droites diminue significativement ce qui implique qu'il existe une chance pour que des modifications minimes de la turbidité, consécutives à une modification de l'intensité de processus de thermisation, aient un effet relativement considérable sur certaines caractéristiques du lait et mettent ainsi en question l'identification du lait U.H.T. 
Pour un degré de turbidité de $50 \mathrm{NTU}$, on peut calculer un certain nombre d'effets chimiques moyens :

- dénaturation des protéines lactosériques :

$<73 \%$

- teneur en HMF :

$<10 \mu \mathrm{mol} / \mathrm{l}$

- perte de lysine disponible :

$<5 \%$

- inhibition d'activité protéolytique :

$>95 \%$

Etant donné que les droites reproduites dans les figures 5 à 7 , aussi bien les valeurs portées en abscisse que celles portées en ordonnée, sont le résultat de réactions chimiques à coefficient de température analogues (Mottar, 1981), on peut supposer qu'un degré croissant de récupération de chaleur ne modifiera pas significativement l'évolution des droites, de sorte que le point d'intersection sera également maintenu aux environs de 50 NTU. Cette supposition est étayée par des observations pratiques. Différentes mesures de la turbidité, de la dénaturation de protéines lactosériques et de la teneur en HMF de laits thermisés dans quatre installations U.H.T. industrielles différentes, à degrés de récupération de chaleur très divers, se situent dans les intervalles de confiance des valeurs individuelles, comme illustré dans les figures 5 et 6 .

\section{CONCLUSION}

Dans le traitement à ultra-haute température du lait, certains processus tels que la destruction de spores et l'inactivation d'enzymes sont désirés pour assurer une conservabilité suffisamment longue du produit. En revanche, il est conseillé, pour des raisons d'ordre organoleptique et/ou nutritionnel, de limiter certaines réactions chimiques, qui se produisent pendant le traitement thermique : la dénaturation de protéines, la réaction de Maillard et la perte de lysine disponible.

Des relations statistiquement significatives ont été constatées entre le degré de turbidité et un certain nombre de caractéristiques importants du lait chauffé, notamment l'effet sporicide, la dénaturation des protéines lactosériques, la formation d'hydroxyméthylfurfural, les pertes de lysine disponible et l'inactivation d'enzymes protéolytiques.

Une turbidité d'environ 50 NTU peut être considérée comme à peu près la valeur-limite d"un lait traité par la méthode U.H.T. indirecte. Une turbidité égale à 50 NTU ou supérieure, dans certaines limites, garantit une qualité acceptable, sans risque d'une efficacité stérilisatrice trop faible.

Il convient de préciser que les essais ont été effectués avec un appareil-pilote, sans récupération de chaleur. En cas d'application d'une récupération de chaleur, il faut s'attendre à des modifications, 
principalement de la relation entre la turbidité et l'effet sporicide. Le degré de récupération de chaleur doit rester limité de façon que l'on obtienne encore une destruction suffisante de spores pour un degré de turbidité d'environ 50 NTU.

\section{Bibliographie}

Agrawala (S.) and Reuter (H.) (1979). - Effect of different temperatures and holding times on whey protein denaturation in a UHT pilot-plant. Milchwiss., 34,735 .

Aschaffenburg (R.) and Drewry (J.) (1959). - New procedure for the routine determination of the various non-casein proteins of milk. 15th Int. Dairy Congress, 3 (5), 1631.

Воотн (V.) (1971). - Determination of FDNB-avaible lysine. A modified Carpenter procedure. J. Sci. Fd. Agric., 22, 658.

Draper (N. R.) and Smith (H.) (1966). - Applied Regression Analysis. J. Wiley, New York.

FInNey (D. J.) (1978). - Statistical Method in Biological Assay. Griffin \& Co, London.

Franklin (J.G.) (1970). - Spores in milk: problems associated with UHTprocessing. J. Appl. Bact., 33, 180.

KeEney (M.) and BasetTe (R.) (1959). - The determination of hydroxymethylfurfural. J. Dairy Sci., 42, 945.

Langeveld (L. P. M.), Robbertsen (T.) en Stadhouders (J.) (1976). - Steriliteitscontrole van aseptisch verpakte produkten. Meded. Ned. Inst. Zuivelonderz., M 11, 3 .

Lechner (E.) (1982). - Analytische Abgrenzung: Pasteurisierte Milch, H.-Milch. Sterilmilch. Dtsche Milchwirtsch., 14, 489.

Mottar (J.) (1981). - Considérations sur la cinétique chimique de l'échauffement du lait à ultra-haute température. Le Lait, 61, 503.

MotTar (J.) et NAudts (M.) (1979), - La qualité du lait chauffé à ultra-haute température comparée à celle du lait pasteurisé et stérilisé dans la bouteille. Le Lait, 59, 476.

MottaR (J.) and Naudts (M.) (1981). - Some observations on différences between UHT milk and in container sterilized milk. IDF Doc., 130, 77.

Noomen (A.) (1975). - Proteolytic activity of milk protease in raw milk pasteurized cow's milk. Neth. Milk Dairy J., 29, 153.

PIEN (J.) (1972). - Méthode permettant de distinguer les laits UHT des autres laits stérilisés. F.I.L., Bulletin annuel, partie V, 57.

WaEs (G.) (1976). - Aerobic mesophilic spores in raw milk. Milchwiss., 31, 521. 